\title{
NUOVE RICERCHE INTORNO AD ALCUNE CLASSI DI GRUPPI DISCONTINUI.
}

\author{
Memoria di Guido Fubini (Catania).
}

Adunanza del 26 novembre 1905.

1. Il riconoscere se un dato gruppo $G$ di trasformazioni in $n$ variabili $(n \supseteq \mathrm{I})$, non contenente operazioni infinitesime opera, o non opera, in modo propriamente discontinuo sui valori reali e complessi di dette variabili, è cosa di grande importanza, non soltanto per le eventuali applicazioni aritmetiche, ma anche per la teoria delle funzioni automorfe; perchè, mentre, per mezzo delle serie di Porncaré e loro generalizzazioni, noi sappiamo costruire funzioni invarianti per un gruppo propriamente discontinuo, non possediamo invece finora alcun metodo per costruire funzioni invarianti per un gruppo impropriamente discontinuo, dato che tali funzioni esistano (naturalmente solo per $n>\mathrm{I}$ ). Di più, la discontinuita deve essere propria, se si vuole che esistano $n$ funzioni indipendenti invarianti per il gruppo.

In alcuni miei lavori *) io mi sono occupato della discontinuita propria dei gruppi, e delle loro applicazioni aritmetiche e funzionali; specialmente in $(A)$ io ho dimostrato la discontinuità propria di molte classi di gruppi, e ho dato un mezzo per riconoscere in generale, se per es. un gruppo proiettivo senza trasformazioni infinitesime è, o non $\grave{e}$, propriamente discontinuo. Ho usato a tal fine di artifici; e noi ci proponiamo di far vedere nelle pagine seguenti che proprio la natura dei nostri gruppi obbliga a ricorrere, per tali questioni, a metodi indiretti. Oltre a questo, gli scopi che si propone il presente lavoro sono i seguenti :

$\mathrm{I}^{\circ}$ Riconoscere se il gruppo delle proiettivita unimodulari a coefficienti interi in $n$ variabili $x$ opera, o no, in modo propriamente discontinuo sui punti reali e complessi

*) Sulla teoria delle forme quadratiche ed Hermitiane, e dei sistemi di tali forme [Atti dell'Accademia Gioenia di Catania, s. IV, vol. XVII (1904)]; Applicazioni analitiche dei gruppi di proiettività, ecc. [Ibid., id.]; Sulle metriche Hermitiane [Atti dell'Istituto Veneto, tomo LXIII (I903-I904), pag. 501]; Sulle funzioni automorfe e iperfuchsiane, ecc. [Annali di Matematica, s. III, t. X (1904), pag. I]; Una questione fondamentale per la teoria, ecc. [Rendiconti della R. Accademia dei Lincei, s. V, vol. XIII (1904), pag. 590]; Sulle metriche Hermitiane [Bollettino dell'Accademia Gioenia di Catania, I905]; Sulla teoria dei gruppi discontinui [Annali di Matematica, s. III, tomo XI (I905), pag. I59].

Io dovrò citare spesso questa ultima Memoria, che richiamerò con la lettera $(A)$; la prima delle Memorie, qui ricordate, sarà citata con la lettera $(B)$. 
dello spazio ambiente (dore cioé le $x$ sono le variabili coordinate): questione, cui già ha accennato il KLEIN nelle sue lezioni.

$2^{\circ}$ Studiare alcuni gruppi, che, o non rientrano dircttamente tra $\mathrm{i}$ tipi studiati nelle mie Memorie citate, o sono di natura affatto distinta, pur avendo una grande importanza per alcuni campi dell'Analisi: tali sono ad es. i gruppi iperabeliani di PICARD, e i gruppi che, nella teoria delle serie $\theta$ in $p$ variabili, hanno l'ufficio che il gruppo modulare compie per la teoria delle funzioni ellittiche *). Di questi gruppi daró pure alcune noteroli generalizzazioni.

$3^{\circ}$ Trattare, almeno nei casi particolari, che più si riconnettono ai precedenti, la questione, che sorge spontanea dalle seguenti considerazioni, e che ha già una grande importanza nella teoria dei gruppi Kleiniani. Sia dato un gruppo $\Gamma$ continuo (per es. il gruppo proiettivo) in $n$ variabili $x_{11}, x_{12}, \ldots, x_{1 n}$. Una sua trasformazione generica sia individuata, quando si diano i punti trasformati di $k$ punti generici. Sia $G$ un gruppo, privo di trasformazioni infinitesime, contenuto in $\Gamma$; e siano $x_{i 1}, x_{i 2}, \ldots, x_{i n}$ $(i=2 . \ldots, b)$ altri $b-\mathrm{I}$ sistemi di variabili. Insieme a ogni trasformazione $T_{\mathrm{t}} \mathrm{di}$ $G$ consideriamo altre $b-\mathrm{I}$ trasformazioni $T_{1}(2 \leq i \leq b)$, che operino sulle $x_{i 1}, \ldots, x_{i n}$, appunto come $T_{1}$ opera sulle $x_{11}, \ldots, x_{1 n}$. Sia $T$ il prodotto delle $T_{1}, \ldots, T_{b}$. E ben evidente che le $T$ generano un gruppo $H$, il quale è certo propriamente discontinuo, se $b \supseteq k$. Si domanda se, e quando, avviene che il gruppo $H$ sia propriamente discontinuo, anche se $b<k$. Per es., siano

$$
x^{\prime}=\frac{\alpha_{r} x+\beta_{r}}{\gamma_{r} x+\delta_{r}}
$$$$
(r=1,2,3, \ldots)
$$

le trasformazioni di un gruppo (Kleiniano) $G$ su una variabile $x$, privo di trasformazioni infinitesime. Il gruppo $\mathbf{r}$ è, nel caso attuale, il gruppo proiettivo a tre parametri (complessi); ed è $k=3$. Ne deduciamo, per quanto abbiamo detto, che in questo caso si puo porre $b \supseteq 3$, ossia che $G$ opera in modo propriamente discontinuo sui sistemi di tre (o più) valori della $x$, e quindi che a ogni gruppo Kleiniano corrispondono funzioni automorfe di tre (o più) variabili indipendenti.

Il KLeIN dice che si puó porre, in tal caso, $b=2$; ma questa asserzione mi sembra dubbia $\left.{ }^{* *}\right)$. La presente questione è in sostanza una generalizzazione del pro-

*) Troveremo una curiosa corrispondenza tra questi gruppi (per $p=2$ ) e i gruppi conformi in una metrica euclidea indefinita: anche queste metriche compariscono cosi nella teoria delle funzioni. Come si vede, questi gruppi offrono particolarità ben distinte da quelle del gruppo modulare: forse per questo non ne è stato ancor fatto uno studio geometrico.

**) Osserviamo infatti, che, com'è ben noto dai lavori di PoINCaré, un tal gruppo Kleiniano $G$ dà origine a un gruppo $G^{\prime}$ di proiettività, trasformante in sè stessa una quadrica del tipo

$$
x_{3}^{2}+x_{2}^{2}+x_{3}^{2}-x_{4}^{2}=0,
$$

e propriamente discontinuo entro la quadrica. Si dovrebbe dimostrare che $G^{\prime}$ opera in modo propriamente discontinuo sulle coppie di punti reali posti sulla quadrica stessa ossia sulle rette, intersecanti 
blema di rivonscere se un dato gruppo í, o non $\dot{i}$, propriamente discontinuo: il che avviene allora, e allora soltanto, che si puó porre $b=\mathrm{I}$. Per quanto questa questione sia qui trattata solo in casi particolari, mi pare opportuno l'avervi accennato esplicitamente in generale, sembrandoni essa di importanza fondamentale per la teoria.

2. Ammessi i teoremi dei $\$ \int_{2}$ e 3 di $(A)$ ridimostrerò qui il primo teorema del $\ 5$ di $(A)$, sia per essere più chiaro e completo, sia perché sulla dimostrazione di questo teorema fondamentale sono modellate in parte alcune delle seguenti considerazioni.

Teorema. - Se un gruppo $G$ di proiettività reali unimodulari, privo di trasformaxioni infinitesine, conserva fisso un sistema $S$ di forme definite di grado qualunque, ossia muta una forma di $S$ in unaltra forma di $S$, allora esso opera in modo propriamente discontinuo sul dato sistema $S$ di forme. (Ricordo che una forma si dice definita, quando ha sempre lo stesso segno, e non si annulla mai per valori reali non contemporaneamente nulli delle variabili, da cui dipende).

Pensiamo le forme di $S$ come punti di uno spazio $\mathbf{\Sigma}$, in cui scegliamo un punto generico $A$, immagine di una forma $*$ di $S$. Poichè le trasformazioni di $G$ non dipendono da parametri variabili con continuità, nessuna trasformazione di $G$ potrà lasciar fisso il punto generico $A$. Se perciò in un intorno di $A$ cadessero infinite coppie di punti equivalenti, esisterebbero in $G$ infinite proiettività, che trasformano una forma prossima ad $\alpha$ in un'altra forma, pure prossima ad $\alpha$. In $G$ esisterebbero dunque $\left[\iint 2\right.$ e 3 di $\left.(A)\right]$ infinite proiettività unimodulari, coi coefficienti minori di una stessa costante, ossia (loc. cit.) $G$ conterrebbe, contro il supposto, proiettività infinitesime.

3. Il PiCARD dimostra nel Journal de Math., I885, per via piuttosto contorta e complicata, che al gruppo aritmetico riproduttore di una forma quadratica del tipo $x_{1}^{2}+x_{2}^{2}-x_{;}^{2}-x_{+}^{2}$, o di una forma riducibile al tipo precedente mediante una proiettività reale, si puó far corrispondere un gruppo di trasformazioni lineari su due varia-

la quadrica in punti reali. Non si puó già concludere di sì, dal fatto che il gruppo opera in modo propriamente discontinuo sui punti interni alla quadrica, perchè lo stesso ragionamento, applicato a una conica $x_{1}^{2}+x_{2}^{2}-x_{3}^{2}=0$ dimostrerebbe che un gruppo fuchsiano, e in particolare il gruppo modulare, opera in modo propriamente discontinuo sulle rette intersecanti la conica in punti reali. E, poichè queste rette, nella teoria di KL.EIN, rappresentano forme di GAUSs indefinite, verrebbe costruita per queste forme una teoria della riduzione affatto analoga alla teoria della riduzione di forme di Gauss definite: cio, che si sa impossibile. E del resto si osservi che il gruppo ciclico generato da una trasformazione lossodromica, la cui componente ellittica è una rotazione di un angolo irrazionale con $2 \pi$, o anihe l'identità, non puó portare un punto interno alla quadrica in un punto infinitamente vicino, ma puó bensi portare una retta in una retta infinitamente vicina: cio basta per escludere che le solite dimostrazioni pernettano di asserire che il nostro gruppo opera sulle rette in modo propriamente discontinuo. $\mathrm{Si}$ puó dire soltanto che esso opera su tali rette in modo semipropriamente discontinuo [cfr. (A), pag. I67]. Nè è lecito quindi fare applicazioni funzionali di un tal gruppo per $b=2$, perchè, come dicemmo, le serie di PoINCARE non sono applicabili che a gruppi propriamente discontinui. 
bili complesse $\xi$, $n$ del tipo:

$$
\xi^{\prime}=\frac{\alpha \xi+\beta}{\gamma \xi+\delta}, \quad n^{\prime}=\frac{a n+b}{c n+d} .
$$

Egli dimostra poi, ricorrendo alla teoria di Hermite della riduzione continua delle forme, che un tal gruppo possiede un campo fondamentale. Con PICARD, noi daremo il nome di "gruppo iperabeliano" a un gruppo siffatto. Questo teorema non è che un caso particolare del seguente teorema più generale, che conduce a nuove classi difunzioni automorfe.

Teorema I.-A ogni gruppo $G$ di collineazioni reali, privo di trasforma ioni infinitesime, che trasformi in sè stessa la forma $V=x_{1}^{2}+x_{2}^{2}-x_{3}^{2}-x_{+}^{2}$ (o una forma riducibile a questo tipo con una collineazione reale) si pui far corrispondere un gruppo propriamente discontinuo iperabeliano $G^{\prime}$ su due variabili complesse $\stackrel{n}{\sharp}$.

Osservazione $\mathrm{I}^{\mathrm{a}}$. - Il presente teorema è una generaliziarione del teorema di $\mathrm{PI}-$ CARD, appunto nello stesso modo che la teoria dei gruppi fuchsiani è una estensione della teoria del gruppo modulare.

Osservazione $\mathrm{II}^{\mathrm{a}}$. - Con procedimenti analoghi a quelli usati nelle mie Memorie cit. $(A)$ e $(B)$, questo teorema si puio estendere ai sistemi di forme $V$, e al gruppo aritmetico riproduttore di una forma $V$ con coefficienti interi in un qualsiasi campo algebrico, reale insieme ai campi coniugati.

Di questo teorema darò due dimostrazioni distinte, che ci condurranno a lor volta a due sue distinte estensioni.

Siano $\xi, n$ i parametri delle generatrici reali e complessi della quadrica $V=0$; il dare una collineazione trasformante $V$ in sè stessa, equivale a definire come essa trasforma le generatrici della $V$. Ora, sostituendo caso mai a $G$ un suo sottogruppo di indice 2, noi possiamo supporre che le trasformazioni di $G$ non scambino tra di loro i due sistemi di generatrici della quadrica $V=0$ : a ogni trasformazione di $G$ verrà cosi a corrispondere una trasformazione del tipo (I). Evidentemente potremo far in modo che le $\alpha, \beta, \gamma, \delta, a, b, c, d$ siano tutte quantità reali. Rappresentiamo ora i valori reali di $\xi(n)$ coi punti reali di una conica $C\left(C^{\prime}\right)$ e i valori complessi di $\xi(n)$ coi punti interni a $C\left(C^{\prime}\right)^{*}$ ). Otterremo cosi che il gruppo in discorso diventa un gruppo, di cui ogni trasformazione è una proiettività mista [cfr. $(A)$, pag. 172), le cui trasformazioni parziali lasciano rispettivamente fisse le coniche $C, C^{\prime}$. Il Teorema $\mathrm{X}$ del $₫ 5$ di $(A)$ ci dà subito la dimostrazione del teorema attuale; il Teorema II del $\ 6$ dimostra che questi gruppi si possono ancora considerare come gruppi di movimenti in una metrica, il cui elemento lineare è somma di due elementi lineari pseudosferici a variabili distinte, per es. $d x_{1}^{2}+e^{-2 x_{1}} d x_{2}^{2}, d y_{1}^{2}+e^{-2 y_{1}} d y_{2}^{2}$. E da ciò si puó trarre un nuovo

*) Il metodo è ben noto: basta ricordare quanto si fa per la rappresentazione proiettiva del gruppo modulare. Cfr. per es. i primi capitoli delle Autormorphe Functionen di KLEIN e Fricke. 
metodo per costruirne i campi fondamentali. Tanto questo teorema, quanto il teorema di Hilbert-Blumenthal, più volte citato in $(A)$, si possono considerare come casi particolari del teorema generale seguente, la cui dimostrazione si compie con mezzi ana$\operatorname{loghi}$ :

Teorema II. - Un gruppo di trasformazioni del tipo

$$
\begin{gathered}
\check{\zeta}_{i}^{\prime}=\frac{\alpha_{i} \dot{\zeta}_{i}+\xi_{i}}{\gamma_{i} \dot{\zeta}_{i}+\dot{\delta}_{i}} \\
\left(\alpha_{i}, \beta_{i}, \gamma_{i}, \delta_{i}\right. \text { essendo quantita reali), }
\end{gathered} \quad(i=\mathrm{I}, 2, \ldots n)
$$

privo di trasformazioni infinitesime, è sempre propriamente discontinuo, e puo servire alla costru-ione di funzioni automorfe coi metodi noti. Un tale gruppo si puo considerare come gruppo di movimenti nella metrica determinata dall'elemento lineare

$$
d s^{2}=\sum_{i}^{n}\left(d x_{i}^{2}+e^{-2 x_{i}} d y_{i}^{2}\right)
$$

A questi gruppi io darei il nome di "gruppi fuchsiani in n variabili».

4. Daremo ora una seconda dimostrazione del Teorema $I$, che ci condurrà ad altri risultati. Le quantità $\zeta, n$ si possono, come è noto, assumere a coordinate dei punti reali e complessi della quadrica $V=0$, definendo un punto di questa quadrica come intersezione delle generatrici di differente sistema, che passano per esso. Il dimostrare percio che $G^{\prime}$ opera in modo propriamente discontinuo sulle variabili complesse $\xi$, n equivale a dimostrare che $G$ opera in modo propriamente discontinuo sui punti complessi della quadrica $V=0$. E, poichè per ogni tal punto passa una e una sola retta reale (la quale incontrerd la quadrica in un altro punto, immaginario coniugato del pri$\mathrm{mo}$ ), ci basterà dimostrare che $G$ opera in modo propriamente discontinuo sulle rette reali, che incontrano in punti complessi la varietà $V=0$. Sia $s_{1}^{2}+s_{2}^{2}=0$ l'equazione della coppia di piani (immaginarii coniugati) tangenti alla $V=0$, e passanti per una di queste rette $r$; sia $s_{3}^{2}+s_{4}^{2}=0$ l'equazione dei piani tangenti alla $V=0$, uscenti dalla retta $r^{\prime}$, polare reciproca di $r$ rispetto alla quadrica $V=0$. Sard

$$
V=k\left(s_{1}^{2}+s_{2}^{2}\right)+l\left(s_{3}^{2}+s_{4}^{2}\right),
$$

dove $k, l$ sono due costanti reali. Una proiettività, che trasformi in sè stessa la forma $V$, e lasci fissa la retta $r$, dovrà lasciare pur fissa la quadrica $V=0$, e quindi anche le quadriche degeneri $s_{1}^{2}+s_{2}^{2}=0, s_{3}^{2}+s_{4}^{2}=0$. Perció le forme $s_{\mathrm{r}}^{2}+s_{2}^{2}, s_{3}^{2}+s_{4}^{2}$ dovranno restar moltiplicate per due fattori costanti: ma, poichè la

$$
V=k\left(s_{1}^{2}+s_{2}^{2}\right)+l\left(s_{3}^{2}+s_{4}^{2}\right)
$$

è trasformata in sè stessa, questi fattori dovranno essere uguali all'unità. In altre parole, anche le forme $s_{1}^{2}+s_{2}^{2}, s_{3}^{2}+s_{4}^{2}$ saranno trasformate in sè stesse. Una proiettività, che porti la retta $r$ in un'altra retta $r^{\prime}$, dovrà portare la coppia di forme corrispondenti alla retta $r$ in un'altra coppia di forme corrispondenti alla $r^{\prime}$. Verra cosi data, per ogni retta $r$, che non incontri la $V=0$, una coppia di forme definite due volte specializzate 
(il cui discriminante è cioè di caratteristical uguale a 2), tali che una proiettività, che lasci fissa la $V$, e porti la rettia $r$ in sè stessa, o in un'altra retta $r^{\prime}$, porterà le forme corrispondenti alla retta $r$ in sè stesse, oppure nelle forme corrispondenti alla retta $r^{\prime}$. E come una retta $r$ determina una coppia di forme, cosi, data una delle nostre coppie di forme, è subito individuata la retta corrispondente. Basterà dunque dimostrare che il gruppo $G$ opera in modo propriamente discontinuo sulle nostre coppie di forme. Ora le due forme $p_{1}, p_{2}$ di una delle nostre coppie di forme sono bensi definite, ma degeneri; è ben evidente però, che noi possiamo scegliere quattro costanti reali $a, b$, $c, d$, tali che le forme $q_{1}=a p_{1}+b p_{2}, q_{2}=c p_{1}+d p_{2}$ siano definite $\mathrm{e}$ non degeneri e che di più $a d-b c \neq 0$. Queste costanti poi si possono supporre non variabili al variare della coppia di forme considerata. Alla coppia di forme $p_{1}, p_{2}$ si è cosi sostituita una coppia $q_{1}, q_{2}$ (di forme definite e a discriminante non nullo), che ancora individua la retta corrispondente. Ci bastera quindi dimostrare che $G$ opera in modo propriamente discontinuo sulle coppie di forme $q_{1}, q_{2}$. Ora il teorema ricordato al $\$ 2$ del presente lavoro non vale soltanto se gli elementi del sistema $S$ sono forme definite, ma anche "a fortiori» se gli elementi del sistema $S$ sono formati da una coppia di forme definite. Resta cosi senz'altro dimostrato di nuovo il Teorema I del $₫ 3$.

Io dico ora che, con un metodo analogo, si puo dimostrare il

Teorema III. - Un gruppo $G$ di collineazioni reali, privo di trasforma ioni infinitesime, che trasforma in sè stessa una forma del tipo

$$
V=x_{1}^{2}+x_{2}^{2}-\left(x_{3}^{2}+\cdots+x_{n}^{2}\right)
$$

(o, ciò che è lo stesso, una forma riducibile al tipo precedente mediante una collineazione reale) opera in modo propriamente discontinuo sui punti complessi della quadrica $V=0$.

Cosi anche le forme quadriche di questo tipo determinano, come i gruppi fuchsiani (la cui teoria è identica, come è noto, a quella dei gruppi proiettivi, che trasformano in sè stessa una conica reale), delle nuove classi di funzioni automorfe!

Per $n=4$, il Teorema III equivale, per quanto dicemmo, al Teorema I; esso è il primo teorema che studii, dal nostro punto di vista, le forme del tipo

$$
x_{1}^{2}+x_{2}^{2}-\left(x_{3}^{2}+\cdots+x_{n}^{2}\right)
$$

per $n \gg 4$. Noi ne vedremo più sotto l'importanza anche per la. teoria della serie $\theta$. Come sopra, basterd dimostrare che $G$ opera in modo propriamente discontinuo sulle rette, che incontrano la $V=0$ in punti immaginarii coniugati. Sia $s_{1}^{2}+\cdots+s_{n-2}^{2}=0$ l'equatione del cono tangente alla $V=o$, avente per nucleo una di queste rette; e sia $s_{n-1}^{2}+s_{n}^{2}=0$ l'equazione della coppia di iperpiani (immaginarii coniugati) passanti per lo spazio a $n-3$ dimensioni, polare reciproco della retta considerata rispetto alla quadrica $V=0$. Basterd, nelle considerazioni precedenti, sostituire le forme $s_{\mathrm{r}}^{2}+\cdots+s_{n-2}^{2}, s_{n-1}^{2}+s_{n}^{2}$ alle forme $s_{\mathrm{I}}^{2}+s_{2}^{2}, s_{3}^{2}+s_{4}^{2}$, perchè venga dimostrato il teorema in discorso. 
Prima di dare ulteriori estensioni del Teorema IIL, ne daremo alcune applicazioni. Supponiamo per il momento $n=5$, ossia supponiamo che lo spazio ambiente $S_{4}$ sia a quattro dimensioni. Da un punto reale $O$ della quadrica escono $\infty^{1}$ rette reali appartenenti alla quadrica e formanti un cono quadrico $l l$. Proiettiamo stereograficamente la quadrica da $O$ sopra un iperpiano $\pi$ a tre dimensioni: sia $\gamma$ la conica reale, intersezione di $W$ con $\pi$. Indichiamo con $x, y, z$ coordinate non omogenee in $\pi$, tali che i piani $x=$ cost., $y=$ cost., $z=$ cost. formino tre fasci, i cui assi $r_{x}, r_{y}, r_{z}$ giacciano sul piano di $\gamma$ e formino un triangolo autopolare rispetto a $\gamma$. L'asse $r_{i}$ sia quello dei tre, che non incontra $\gamma$ in punti reali. Alle proiettività in $S_{4}$, che lasciano fissa la $V=o$ corrisponde in $\pi$ il gruppo delle trasformazioni, che formano il gruppo conforme per la metrica euclidea determinata dalla conica $\gamma ;$ scelta come assoluto $(o$, in altre parole, che formano il gruppo che muta in sè stessa l'equazione $d x^{2}+d y^{2}-d z^{2}=0$, $o$, in altre parole ancora, che moltiplicano per un fattore finito la forma differenziale $\left.d x^{2}+d y^{2}-d \tau^{2}\right)^{*}$ ). Otteniamo cosi il

Teorema IV. - Un gruppo $G$ di trasformazioni reali, conformi per una metrica euclidea a tre dimensioni indefinita (il cui assoluto è cioè una conica reale, o, ciò the è lo stesso, il cui elemento lineare è la forma differenziale $d x^{2}+d y^{2}-d z^{2}$, oppure una forma, riducibile a questa mediante un cambiamento di variabili coordinate) $e$ privo di trasformazioni infinitesime opera in modo propriamente discontinuo sui punti complessi dello spazio ambiente.

A tali gruppi, potremo, per quanto si dird più sotto, dare il nome di gruppi Hermitiani.

E poi naturale che, ponendo nel Teorema III $n>5$, si trova:

TeOREma V.-Il precedente risultato vale per ogni metrica euclidea indefinita, il cui elemento lineare sia del tipo $-d x_{1}^{2}+d x_{2}^{2}+\cdots+d x_{n}^{2}$, anche se $n>3$.

L'importanza del Teorema IV sta massimamente in questo che, come vedremo, il gruppo di HeRmite (Comptes Rendus, I855) delle trasformazioni di grado i delle serie $\theta$ in due variabili è un caso particolare dei gruppi ivi considerati; cosicché detto teorema dà a siffatto gruppo una generalizzazione analoga a quella, che la teoria dei gruppi fuchsiani dil per il gruppo modulare.

Il PICard (loc. cit.) e più tardi i suoi allievi hanno bensi studiato il citato gruppo di Hermite, ma limitandosi a dimostrare la discontinuita propria e dare quindi le applicazioni funzionali di un suo sottogruppo, il quale era poi un gruppo iperabeliano. Questo sottogruppo e gruppi analoghi si otterrebbero dal Teorema III (per $n=5$ ), se noi considerassimo quei gruppi, che, oltre a lasciar fissa la $V=0$, mutassero in sè stesso anche l'iperpiano $x_{5}=0$. Otterremo cosi i gruppi riproduttori di una forma

*) Ciò è bene evidente per chi ricordi il noto rapporto, che passa tra il gruppo conforme dell'ordinario spazio euclideo e il gruppo delle proiettivita che trasformano in sè stessa una quadrica dello spazio a quattro dimensioni. 
$x_{1}^{2}+x_{2}^{2}-x_{3}^{2}-x_{4}^{2}$, di cui già parlammo al Teorema $\mathrm{I}$, e che non darebbero quindi nulla di nuovo. E poi opportuno osservare:

$I^{\circ}$ Oltre al citato gruppo di Hermite, noi siamo gia in grado di determinare infiniti altri gruppi Hermitiani: p. es. i gruppi aritmetici riproduttori di una forma a coefficienti interi, riducibile con una proiettiviti reale al tipo $x_{1}^{2}+x_{2}^{2}-\left(x_{3}^{2}+x_{4}^{2}+x_{5}^{2}\right)$.

$2^{\circ}$ Il Teorema III si puó estendere ${ }^{*}$ ) anche ai sistemi di forme quadratiche del tipo anzidetto, e quindi anche al gruppo aritmetico riproduttore di una delle nostre forme, a coefficienti interi in un qualsiasi campo algebrico, reale insieme ai campi coniugati: e cosi si ottengono nuove classi di gruppi propriamente discontinui, capaci di applicazioni funzionali !

Di un'analoga generalizzazione sono suscettibili $\mathrm{j}$ Teoremi IV e V, e io qui la enuncierò brevemente:

Teorema VI. - Sia dato un gruppo di trasformazioni $T$, privo di trasformazioni infinitesime, in $n_{1}+n_{2}+\cdots+n_{k}$ varialili $x_{i l}(i=\mathrm{I}, 2, \ldots, k ; l=\mathrm{I}, 2, \ldots, n)$. Ogni trasformazione $T$ sia il prodotto di pii trasformazioni $T_{i}(i \leq k)$ conformi per la metrica euclidea indefinita, determinata dall'elemento lineare $-d x_{i 1}^{2}+\sum_{i=2}^{n_{i}} d x_{i l}^{2}$. Il gruppo $G$ opera in modo propriamente discontinuo sui punti reali e complessi dello spazio, in cui le $x$ sono variabili coordinate non omogenee.

Dimostriamo ora che il gruppo di Hermite, citato più sopra, non è che un caso particolare dei gruppi Hermitiani, cui si riferisce il Teorema IV. Sopra una superficie Riemanniana di genere 2 siano $\omega_{1}, \omega_{2}, \omega_{3}, \omega_{4} \mathrm{e} \omega_{1}^{\prime}, \omega_{2}^{\prime}, \omega_{3}^{\prime}, \omega_{4}^{\prime}$ i periodi di due integrali abeliani di prima specie; posto $\omega_{i k}=\omega_{i} \omega_{k}^{\prime}-\omega_{k} \omega_{i}^{\prime}$, è, com'è noto,

$$
\omega_{i k}+\omega_{k i}=(1)_{12}^{(1)} ;+4+\omega_{1 ;} \omega_{t^{2}}+\omega_{14}^{()_{23}}=0 \text {. }
$$

Di più, per le relazioni, che legano detti periodi, sarà:

$$
\omega_{13}+\omega_{2+}=0 \text {. }
$$

Poichè $\omega_{i k}=-\left(\omega_{k i}\right.$, potremo, d'ora in poi, limitarci a considerare quelle $\omega_{i k}$, per cui $i<k$. Le $\omega_{i k}$ si potranno così considerare, com'è noto, quali coordinate omogenee dei punti di uno spazio a 5 dimensioni, posti sull'intersezione $V$ dell'iperpiano $\omega_{12}+\omega_{3-4}=0$ e della quadrica $\omega_{12}()_{3+}-\omega_{1 ;} \omega_{2+}+\omega_{1+} \omega_{23}=0$. Questo iperpiano è uno spazio lineare a 4 dimensioni, in cui potremo assumere come coordinate $x_{i}(i \leq 5)$ le $\omega_{12}, \omega_{13}, \omega_{14}$, $\omega_{23}, \omega_{24}$. La $V$ sard così data dall'equazione $x_{1} x_{2}+x_{3} x_{4}-x_{3}^{2}=0$ : essa è quindi una quadrica del tipo da noi studiato. Ora il gruppo di Hermite rappresenta l'effetto prodotto da un cambiamento dei tagli, the rendono semplicemente connessa la superficie di RiEMANN : un tale cambiamento dà luogo, com'è noto, a una trasformazione lineare

*) Con gli stessi metodi usati in $(A)$ e $(B)$ per estendere ai sistemi di forme e a campi algebrici qualunque la teoria dei gruppi riproduttori di una forma quadratica del tipo $x_{\mathrm{I}}^{2}+\ldots+x_{n-1}^{2}+x_{n}^{2}$, di una forma Hermitiana, ecc. 
sulle $\omega_{i}$, e conseguentemente anche sulle $\omega_{i h}$, che lascia però fissa la nostra quadrica $V$. Se noi ora dalle $\omega_{i}$ passiamo alla considerazione dei moduli, ossia dei periodi $\tau_{11}$, $\tau_{12}=\tau_{21}, \tau_{22}$ degli integrali normali di prima specie, facciamo appunto una trasformazione, che corrisponde alla proiezione stereografica da noi usata per stabilire il Teorema IV; e il gruppo di Hermite diventa cosi un gruppo conforme in una metrica euclidea indefinita [determinata da una conica assoluto reale, cui sono tangenti ${ }^{*}$ ) i piani $\tau_{\mathrm{i} 1}=$ cost. in un punto $A$, i piani $\tau_{22}=$ cost. in un punto $B$, e che è incontrata dai piani $\tau_{\mathrm{r} 2}=$ cost. precisamente nei punti $\left.A, B\right]$.

Il Teorema IV ne dimostra quindi la discontinuita propria, e permette che gli si applichi la teoria delle serie di PoINcaré generalizzate. La costruzione effettiva del campo fondamentale è peró certamente cosa complicata e difficile, per quanto la conoscenza delle sostituzioni generatrici del gruppo (cfr. per es. KRAzER, Theorie der Thetareiben) e il fatto che due punti complessi hanno due invarianti ${ }^{* *}$ ) rispetto al gruppo conforme in una metrica euclidea indefinita possano facilitarne lo studio teorico.

Un metodo indiretto per costruire i campi fondamentali di questo, e degli altri gruppi, studiati nel presente lavoro, si puó ottenere, ricordando che tutti questi gruppi si possono considerare come gruppi proiettivi, trasformanti in sè stessa una quadrica $V$, e limitandoci poi a studiare, come essi trasformano i punti complessi di $V$. Ora a un punto complesso di $V$ si puó far corrispondere, come abbiamo visto più sopra, una coppia di forme $p_{1}, p_{2}$ tali che si possono scegliere in infiniti modi due costanti $\lambda, \mu$ in guisa che $\lambda p_{1}+\mu p_{2}$ sia una forma positiva a discriminante non nullo.

Nello spazio $\Sigma$, i cui punti corrispondono biunivocamente alle forme quadriche nello spazio ambiente, queste forme $\lambda p_{1}+\mu p_{2}$ determinano una varietà subordinata $\Sigma_{1}$. Ora noi sappiamo (cfr. $A$ ) costruire in $\Sigma$ un campo fondamentale $P$ del nostro gruppo: quei punti complessi di $V$, tali che il loro punto corrispondente di $\Sigma_{1}$ è interno a $P$, costituiscono un campo, che si puó assumere come campo fondamentale del nostro gruppo sulla quadrica $V$.

Per la teoria delle serie $\theta$ in più di due variabili indipendenti, non sono riuscito a dimostrare, con mezzi analoghi a quelli fin qui usati, la discontinuità propria del gruppo delle trasformazioni di grado $\mathrm{I}$ : questa discontinuità puó risultare dalle formole, che dànno l'effetto di queste trasformazioni sulle serie $\theta$. Ma una tale dimostrazione non porterebbe ad alcuna notevole generalizzazione, appunto come la dimostra-

*) L'equazione di questa conica, in coordinate omogenee, e $\tau_{11} \tau_{22}-\tau_{12}^{2}=0$.

**) Infatti basta ricordare che due rette, poste nello $S_{4}$, in cui esiste la quadrica

$$
x_{1}^{2}+x_{2}^{2}-\left(x_{3}^{2}+x_{4}^{2}+x_{5}^{2}\right)=0
$$

hanno due invarianti nella metrica (indefinita) determinata da questa quadrica : la loro minima e massima distanza. Questi invarianti tengono nel caso attuale il luogo, che la distanza di due punti ha nella teoria dei gruppi di movimenti $[\mathrm{cfr} .(A)]$ e potrebbero essere utili alla costruzione dei campi fondamentali. 
zione della discontinuita propria del gruppo modulare, dedotta dalla teoria dell'invariante assoluto delle funzioni ellittiche, non puó condurre alla teoria delle funzioni fuchsiane. Ecco qui dunque un campo, ancora inesplorato, di ricerche, che può condurre a risultati interessanti chi l'affronti con forze migliori delle mie.

Una ulteriore estensione dei Teoremi I, III, IV conduce a nuove classi di gruppi propriamente discontinui su variabili complesse e tratta in un caso particolare la terza questione, cui abbiamo accennato al $\ \mathrm{I}$.

Teorema VII. - Un gruppo $G$ di proiettività reali, di cui nessuna infinitesima, che trasformi in sè stessa una forma quadrica del tipo

$$
V=\sum_{\mathrm{r}}^{k} x_{i}^{2}-\sum_{k+\mathrm{r}}^{n} x_{i}^{2} \quad(k<n-\mathrm{I}),
$$

opera in modo propriamente discontinuo sugli spazii lineari reali a $k-\mathrm{I}$ o a $n-k-\mathbf{I}$ dimensioni, contenuti nello spazio ambiente, e non aventi punti reali comuni con la $V=0$.

Un tale spazio $S$, e lo spazio polare $S^{\prime}$ determinano infatti una coppia di forme quadratiche (degeneri) definite, le quali, uguagliate a zero, dànno l'equazione dei coni tangenti alla $V=0$, aventi per nucleo rispettivamente gli spazii $S, S^{\prime}$ suddetti.

E a una tale coppia di forme si possono applicare quelle considerazioni, che gia usammo per le forme $s_{1}^{2}+s_{2}^{2}, s_{3}^{2}+s_{+}^{2}$, quando dimostrammo il Teorema I. Sia ora $b$ per es. il minore dei due numeri $k-\mathrm{I}, n-k-\mathrm{I}$; supponiamo $b$ dispari (risultati analoghi si hanno per $b$ pari). Notiamo che uno spazio $S_{h}$ ad $b$ dimensioni è determinato da $b+$ I suoi punti generici, i quali si possono anche scegliere sull'intersezione di $S_{b}$ con la quadrica $V=0$. Se $S_{b}$ non taglia la $V=0$ in punti reali, questi $b+$ I punti, posti sulla citata intersezione, si possono scegliere a due a due immaginarii coniugati, cosicchè dal teorema precedente si deduce a fortiori che:

Teorema VII ${ }^{\mathrm{bis}}$. - Il gruppo $G$ del Teorena VII opera in modo propriamente discontinuo sui sistemi $d i \frac{b+\mathrm{I}}{2}$ punti complessi posti sulla $V=0$.

E, proiettando stereograficamente la $V=o$ da un suo punto reale su un iperpiano reale, avremo, con le stesse notazioni:

TEOREMa VIII. - Un gruppo di trasformazioni reali, conformi per la metrica euclidea indefinita, determinata dall'elemento lineare

$$
\left(d y_{1}^{2}+d y_{2}^{2}+\cdots+d y_{k}^{2}\right)-\left(d y_{k+1}^{2}+\cdots+d y_{n-2}^{2}\right)
$$

opera in modo propriamente discontinuo sui gruppi di $t$ punti complessi dello spazio ambiente se $t \supseteq \frac{b+\mathbf{I}}{2}$.

A tali gruppi potremmo dare il nome di gruppi iperhermitiani.

Osservazione. - Anche questo Teorema è suscettibile di una estensione analoga a quella che il Teorema VI dà dei Teoremi IV e V.

5. Tratterò ora della prima delle questioni proposteci al $\ \mathrm{I}$, rispondendo alla domanda, gid posta dal KLEIN : 
Il gruppo $G$ di trasformazioni lineari unimodulari, a coefficienti interi su nu variabili $x_{i}(\mathrm{I} \leq i \leq n)$ opera, o no, in modo propriamente discontinuo sui punti complessi dello spazio ambiente?

Basterd applicare al caso attuale il metodo esposto al $\ 8$ di $A$. Per fissare le idee supponiamo $n=4$, e scriviamo ogni forma definita sotto la forma:

$\mu_{1}\left(x_{1}+\varepsilon_{1} x_{2}+\varepsilon_{2} x_{3}+\varepsilon_{3} x_{4}\right)^{2}+\mu_{2}\left(x_{2}+\varepsilon_{4} x_{3}+\varepsilon_{5} x_{4}\right)^{2}+\mu_{3}\left(x_{3}+\varepsilon_{6} x_{4}\right)^{2}+\mu_{4} x_{4}^{2}$, dove le $\mu_{i}$ sono positive. Una tal forma si puó dire ridotta, se

$$
2 \mu_{2} \supseteq \mu_{1}, 2 \mu_{3} \supseteq \mu_{2}, 2 \mu_{4} \supseteq \mu_{3},\left|\varepsilon_{i}\right| \leq \frac{x}{2}, \quad\left(|\varepsilon|_{i}=\text { valore assoluto di } \varepsilon_{i}\right) .
$$

Nello spazio $S_{9}$ a nove dimensioni, i cui punti corrispondono biunivocamente alle forme quadratiche nelle variabili $x_{i}{ }^{*}$ ), queste disuguaglianze servono a limitare un poliedro $P$, che si può considerare come il campo fondamentale del nostro gruppo aritmetico $G$ nello spazio $S_{9}$. Questo poliedro $P$, e i campi equivalenti riempiono tutta quella regione $R$ di $S$, che corrisponde a forme definite. Tra le varietà limiti di $R$ esiste la varietà $W$, che corrisponde alle forme quadriche spezzate in due forme lineari immaginarie coniugate. Ora nessun pezzo a sei dimensioni della $W$ fa parte del contorno di $P$ : ciò che basta per concludere, per i risultati di $(A)$, che il gruppo $G$ non opera in modo propriamente discontinuo sui piani complessi dello spazio, in cui le $x_{i}$ sono coordinate omogenee di punto. Altrettanto si dimostra dualmente per i punti di detto spazio. E si trae gid da questo esempio la conclusione che l'aver ricorso, come è fatto in $(A)$, a mezzi indiretti per studiare i gruppi proiettivi (senza trasformazioni infinitesime) non è già dovuto a imperfezione di metodo, ma alla natura stessa di tali gruppi.

Catania, 21 novembre I905.

Guido Fubini.

*) Al solito, non consideriamo come distinti due punti, le cui forme corrispondenti differiscono soltanto per un fattore. 